\title{
BMJ Open Evidence of past and current collaborations between traditional health practitioners and biomedical health practitioners: a scoping review protocol
}

\author{
Ngcwalisa Amanda Jama (D) , Anam Nyembezi, Uta Lehmann
}

To cite: Jama NA, Nyembezi A, Lehmann U. Evidence of past and current collaborations between traditional health practitioners and biomedical health practitioners: a scoping review protocol. BMJ Open 2021;11:e043452. doi:10.1136/ bmjopen-2020-043452

- Prepublication history for this paper is available online. To view these files, please visit the journal online (http://dx.doi. org/10.1136/bmjopen-2020043452).

Received 04 August 2020 Revised 25 November 2020 Accepted 27 November 2020

Check for updates

(C) Author(s) (or their employer(s)) 2021. Re-use permitted under CC BY-NC. No commercial re-use. See rights and permissions. Published by BMJ.

School of Public health, University of the Western Cape, Cape Town, Western Cape, South Africa

Correspondence to Ngcwalisa Amanda Jama; ngcwalisaj@gmail.com

\section{ABSTRACT}

Introduction Healthcare seekers around the globe use more than one healthcare system, with most using the traditional and the Western approaches concurrently. To date, little collaboration between the two systems has taken place within the mental health space compared with other areas of medicine. In order to inform integrating plans for traditional health practitioners and biomedical health practitioners in the South African mental health system, it is important to know which models of collaboration are used in other medical settings and contexts. This study aims to document global evidence on collaboration practices between traditional health practitioners and biomedical professionals when working with various health conditions.

Methods and analysis This scoping review will be guided by an improved Arksey and 0'Malley framework, the 2010 Levac et al methodological framework and the 2017 Joanna Briggs Institute guidelines. A systematic literature search will be carried out using seven different databases, EMBASE, PubMed, LILACS MEDLINE, APA PsycArticles, CINAHL Plus, Academic Search Complete and Scopus, in addition to the WHO repository, bibliographical search engines, and Open Access Theses and Dissertations. Moreover, the references of relevant publications between January 1978 and March 2020 will be scanned. Two reviewers will independently screen articles for eligibility based on the predetermined inclusion and exclusion criteria. Thematic analysis and descriptive numerical analysis will be performed using ATLAS.ti V.8 and Excel software, respectively. The results for this review will be presented using the Preferred Reporting Items for Systematic Reviews and Meta-Analysis: Extension for Scoping Review.

Ethics and dissemination This study will not require ethics approval because publicly available material will be used. Study findings will be published in an open-access journal and be presented to other key health system stakeholders and academic research gatherings.

\section{INTRODUCTION}

Traditional health practitioners (THPs) and biomedical health practitioners (BHPs) have practised concurrently in the healthcare system for a long time, with the Westerntrained healers being recognised as the
Strength and limitations of this study

- This will be the first scoping review that maps evidence of past and current practitioner-level collaborations between traditional health practitioners and biomedical health practitioners.

- The search strategy will be conducted on seven databases, and a literature search will be inclusive of grey literature from government and institutional libraries.

- The Rayyan software that will be used for screening data in the study allows for 'blind' screening, which will ensure minimisation of bias. This will further increase the credibility of the screening process.

- Although inclusive of global evidence, this review will only review articles written in English due to the lack of resources for data translation.

- The reviewers will not conduct a formal appraisal of the included studies and, therefore, there is a possibility of including studies with methodological limitations. However, to limit the inclusion of studies with poor quality, only peer-reviewed and published primary studies will be included.

official health providers in most health policies around the world. ${ }^{1-3}$ A WHO expert meeting on mental health in 1978 reported that collaboration between THPs and Western-trained healers is needed since THPs are in an ideal position to influence the attitudes of healthcare seekers. ${ }^{4}$ Besides the inevitable need to increase human resources for poorly resourced and developing countries, the call was made by WHO for THPs and BHPs to work together in offering comprehensive and holistic healthcare to the pluralistic populations that use both healthcare models. $^{5-7}$

Multisectoral collaboration is viewed as essential for elevating the burden on the health system, and it is also regarded as a necessary step towards achieving an integrated health system. ${ }^{8}$ Since the 1978 Declaration of Alma Ata by WHO, the recognition given to THPs 
in all government laws and regulations is widespread across most countries, ${ }^{29}$ with the African and Southeast Asian countries leading in terms of having programmes for the integration of THPs into official health services. ${ }^{9}$ Despite the progress made, the implementation of these policies and the advancement of programmes has been slow. In 1982, Lee ${ }^{10}$ followed by Pinkoane et $a l^{11}$ in the early 2000s and Busia and Kasilo ${ }^{12}$ in 2010, summarised the different ways in which countries have attempted to integrate traditional medicine and THPs into the health sector, and their findings illustrate that there are few countries with an integrated health system.

The lack of official frameworks for collaboration between THPs and biomedical professionals together with other governmental and interpersonal hindering factors has been cited as the cause of this. ${ }^{113-16}$ Interestingly, with the changing landscape of the health system due to the recent pandemics that have added stress to the primary health system and advanced policy and research in regard to traditional medicine, several collaboration projects have emerged. This has mainly occurred in the management of HIV and AIDS in Africa. ${ }^{517} 18$ Current evidence in South Africa suggests that more THPs and BHPs are willing to forge collaborations at the service delivery level. ${ }^{19}{ }^{20}$ However, there is a paucity of research on effective models of collaboration and the settings and context in which they can be applied.

In order to accelerate the rate of collaboration, it is vital to learn from previous attempts at establishing collaborations between THPs and BHPs. This review attempts to map existing evidence on practitioner-level collaborations for all health conditions. To date, there is a scarcity of studies aimed at consolidating evidence on these projects. As a result, there is little understanding of how to implement and sustain collaboration projects better. The review forms part of the first phase of a doctoral study on collaboration between THPs and mental health practitioners in South Africa. As such, results from the current study will be used to inform upcoming research on collaboration models between THPs and mental health practitioners within the South African context.

\section{OBJECTIVE}

The main objective of this scoping review is to systematically map global evidence on collaborations between THPs and biomedical professionals' practices when working on any health conditions and to identify possible knowledge gaps.

\section{METHODS AND ANALYSIS \\ Protocol design}

According to Davis et $a l^{21}$ a scoping review is instrumental in providing conceptual clarity about a certain field of evidence. A scoping review was considered suitable for investigating the type and depth of evidence that is available regarding collaborations between THPs and
BHPs. This scoping review will be guided by Arksey and O'Malley, ${ }^{22}$ the methodological framework of Levac et $a l^{23}$ and the 2017 recommendations of the Joanna Briggs Institute (JBI) ${ }^{24}$ The methodological framework that guided this review involved five stages: (I) identifying the research question; (II) identifying relevant studies; (III) study selection; (IV) charting the data; (V) collating, summarising, and reporting the results.

\section{Stage I: identification of the research question}

The study will use the $\mathrm{JBI}^{24}$ PCC mnemonic (population, concept and context) to determine the eligibility of the research question, as presented in table 1. Due to the diverse nature of traditional practices, a clear definition with set parameters for this study is provided in the following section.

\section{Research question}

The key research question for this review study is as follows: What is the global evidence of past and current collaboration practices between THPs and biomedical professionals when working on any health conditions?

\section{Population term definition}

$\mathrm{WHO}^{9}$ defines THPs as practitioners who use indigenous or native approaches, knowledge, spiritual therapies and beliefs that incorporate plant, animal and/or mineralbased medicines separately or in combination to maintain well-being. In this study, the practice of incorporating spirituality during healing is key in distinguishing THPs.

$\mathrm{WHO}^{9}$ defines BHPs as Western-trained health professionals who use the principles of biology or biochemistry. ${ }^{9}$

\section{Stage II: identification of relevant literature}

A systematic search will be conducted on the following databases: EMBASE, PubMed and EBSCOhost interface, and will include MEDLINE, APA PsycArticles, Cumulative Index of Nursing and Allied Health (CINAHL) Plus, Academic Search Complete and Scopus. To capture Latin American and Caribbean health sciences literature, the reviewers will also search the LILACS database. In addition, a literature search will be conducted using free-text words on bibliographical search engines such as Google Scholar and Academia.edu. National health departments, the WHO repository, and the Open Access Theses and Dissertations library will be searched for grey literature such as reports, dissertations and theses. The search will include studies conducted between January 1978 and March 2020 that were published in English.

The search process will be iterative and will be documented in the methods section of the scoping review report. During the search period, the 'MeSH term', the title/abstract tab, the text word tab and the keyword tabs will be chosen. The search terms will be combined using the Boolean (AND, OR) method. A preliminary search was conducted on Google and Google Scholar to explore terms that are used to refer to Western and traditionally trained healers in the global and local literature. This assisted in the choice of the terms that will be used in 
Table 1 PCC framework and eligibility criteria for inclusion and exclusion of studies

\begin{tabular}{|c|c|c|c|}
\hline Criteria & Determinants & Inclusion criteria & Exclusion criteria \\
\hline Population & $\begin{array}{l}\text { THPs: THPs vary across } \\
\text { countries and tribes } \\
\text { BHPs: All relevant BHPs } \\
\text { All participants are older than } \\
18 \text { years }\end{array}$ & $\begin{array}{l}\text { Articles reporting on } \\
\text { collaboration with THPs who } \\
\text { are classified as traditional } \\
\text { healers, diviners and herbalists, } \\
\text { alternative healers, native } \\
\text { healers, aboriginal healers, } \\
\text { indigenous healers, traditional } \\
\text { Chinese healers, Traditional } \\
\text { Indian healers, Shammas } \\
\text { Articles reporting on different } \\
\text { cadres of BHPs such as } \\
\text { biomedical healers, general } \\
\text { practitioner, psychologists, } \\
\text { psychiatrists, medical doctors, } \\
\text { nurses, medical doctors, } \\
\text { physicians, healthcare workers, } \\
\text { social workers }\end{array}$ & $\begin{array}{l}\text { Articles presenting evidence on } \\
\text { collaboration with faith healers, } \\
\text { traditional surgeons, traditional } \\
\text { birth attendants, midwives, } \\
\text { doulas }\end{array}$ \\
\hline Content & $\begin{array}{l}\text { Collaboration practices such } \\
\text { as task-shifting, consultation, } \\
\text { cooperation and integration of } \\
\text { services } \\
\text { Medical conditions such as } \\
\text { mental health, HIV and AIDS, } \\
\text { TB, cancer, and mild or chronic } \\
\text { illnesses }\end{array}$ & $\begin{array}{l}\text { Evidence presenting one or } \\
\text { more forms of collaboration at } \\
\text { all levels of the continuum of } \\
\text { care } \\
\text { Studies focusing on all health } \\
\text { conditions }\end{array}$ & $\begin{array}{l}\text { Articles and studies that do not } \\
\text { include collaboration between } \\
\text { practitioners } \\
\text { Studies focusing on animals }\end{array}$ \\
\hline Context & Global & $\begin{array}{l}\text { Articles published between } \\
\text { January } 1978^{\star} \text { and March } 2020 \\
\text { reporting global evidence }\end{array}$ & $\begin{array}{l}\text { Evidence published in non- } \\
\text { English languages }\end{array}$ \\
\hline
\end{tabular}

${ }^{*}$ The year 1978 was chosen because it is the year WHO declared the importance of THPs and traditional medicine in the 1978 Declaration of Alma Ata.

†Not part of the JBI PCC framework. Added by the authors to highlight included and excluded evidence types.

$\mathrm{BHP}$, biomedical health practitioner; THP, traditional health practitioner.

the review. The search strategy is presented in the second column of table 2. With the assistance of an experienced librarian, a pilot search of the search terms was conducted in PubMed, and this is presented in the electronic search record table below. The inclusion and exclusion criteria defined in table 1 were applied during the pilot search and will be applied in the literature search.

\section{Pilot search}

The electronic search record and results of the pilot search are presented in table 2.

An updated record of searches will be kept to monitor when the same search terms are applied in other databases (table 2). The literature results will be exported to EndNote X7 software. At this stage, all duplicated articles will be excluded.

\section{Stage III: study selection}

The study selection process is shown in the PRISMA flow diagram (figure 1).

Screening for this study will be conducted at two levels. The first level will involve double and independent screening of the title and the abstract based on whether they potentially meet the inclusion criteria described in table 1. The second level will involve full-text screening, which will be conducted independently by two screeners. Should the full texts not be available online, the authors will be contacted to request the full texts. In cases where such attempts fail, the abstracts will be excluded from the study. Screening at both levels will be performed on Rayyan QCRI Systematic Reviews Web Application. ${ }^{25}$ This process will be blinded to minimise bias. The screening manual, which comprises instructions and eligibility form 
Table 2 Electronic search record and results of the pilot search

\begin{tabular}{|c|c|c|c|}
\hline $15 / 10 / 2020$ & $\begin{array}{l}(((((((((((((((((((((((((\text { Traditional health } \\
\text { practitioners(Text Word)) OR (Traditional } \\
\text { healers(Text Word))) OR (“aboriginal } \\
\text { healers"(Text Word))) OR (alternative healers } \\
\text { [Text word))) OR (“indigenous healers"(Text } \\
\text { Word))) OR (diviners(Text Word))) OR } \\
\text { (herbalists(Text Word))) OR (traditional Chinese } \\
\text { healers(Text Word))) OR (Traditional indian } \\
\text { healers(Text Word))) OR (shammas(Text Word))) } \\
\text { AND (biomedical health practitioners(Text } \\
\text { Word))) OR (biomedical healers(Text Word))) } \\
\text { OR (psychologists(MeSH Terms))) OR } \\
\text { (psychiatrists(MeSH Terms))) OR (“medical } \\
\text { doctors"(Text Word))) OR (nurse(Text Word))) OR } \\
\text { (“social workers"(Text Word))) OR (“healthcare } \\
\text { workers"(Text Word))) OR (doctor(Text Word))) } \\
\text { OR (physician(Text Word))) OR (“medical } \\
\text { staff”(Text Word))) OR (“general practitioner"(Text } \\
\text { Word))) AND (collaboration(Text Word))) OR } \\
\text { (“task shifting"(Text Word))) OR (integration(Text } \\
\text { Word))) OR (cooperation(Text Word))) NOT (birth } \\
\text { attendants, traditional(MeSH Terms))) NOT } \\
\text { (midwives(MeSH Terms))) NOT (doulas(MeSH } \\
\text { Terms))) NOT (“traditional surgeons"(Text Word)) } \\
\text { Filters applied: Journal Article, English, } \\
\text { Complementary Medicine, from 1978/1/1 to } \\
2020 / 3 / 30 .\end{array}$ & PubMed & 6872 \\
\hline
\end{tabular}

to guide screeners, is presented in online supplemental appendices $\mathrm{A}$ and $\mathrm{B}$. This guide will be trialled on the abstract screening of 10 articles and full-text screening

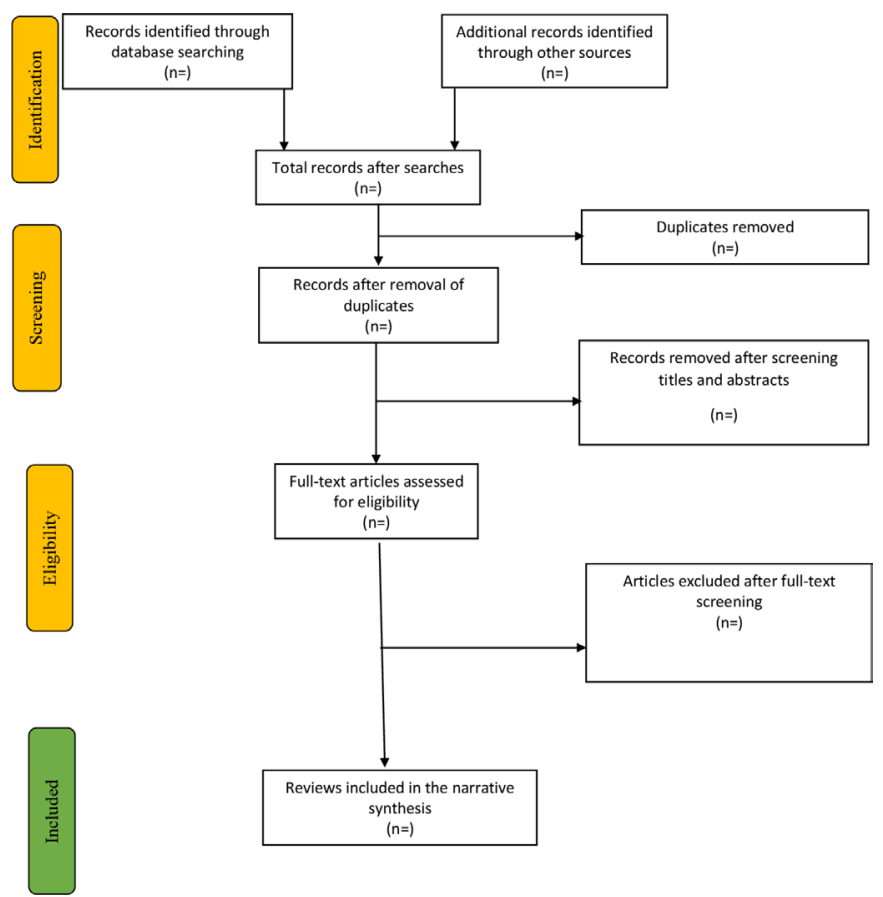

Figure 1 PRISMA flow diagram. of 4 articles. The two reviewers will meet to resolve any conflicts recorded on the Rayyan application, and study supervisors will be consulted to reach a consensus regarding unresolved conflicts.

\section{Stage IV: charting}

Data will be charted independently by two researchers. For this study, a data collection Excel worksheet will be used. This will be adapted from the $\mathrm{JBI}^{24}$ data charting document (see online supplemental appendix $\mathrm{C}$ ) to chart the bibliographic details of the study, the study design, the aim of the study, the characteristics of the THPs and BHPs, the intervention processes, the conclusions for the primary and the secondary outcomes of the interventions, and other aspects. The charting tool will be piloted on a random selection of two to five articles to determine if the reviewers have a full grasp of the data collection instructions and whether the tool is comprehensive enough to capture relevant information. After the pilot study, the team will update the charting sheet to accommodate emerging items.

\section{Stage V: collating, summarising and reporting results}

For this scoping review, the models of collaboration between the THPs and the BHPs that are identified and extracted will be coded and analysed using a thematic analysis technique. Data will be coded by two researchers 
independently, employing a qualitative computer-based software, ATLAS.ti V.8, and Excel software. The new Preferred Reporting Items for Systematic Reviews and Meta-Analysis: Extension for Scoping Review guide will be used to report outcomes of the review. ${ }^{26}$

The analysis will highlight the nature and the distribution of the studies that are included in the review. Tables and chart maps that illustrate the distribution of the reviewed studies geographically and the nature of the collaborations between the THPs and the BHPs will be produced. In addition, a narrative account of the generated themes surrounding the type of collaborations and their outcomes will be presented. To minimise bias and ensure a consistent approach in reporting the results, the research team will meet to discuss the emerging themes. Furthermore, the study team will scrutinise the meanings of the findings as they relate to the overall aim of the study and discuss the implications for future research, practice and policy.

\section{Patient and public involvement}

This study will use publicly available data; therefore, patients or the public will not be involved in the design, or conduct, or reporting, or dissemination plans of this review.

\section{ETHICS AND DISSEMINATION}

This study will constitute the first step in a multiphase research doctoral study aimed at investigating emerging small-scale collaboration models between THPs and BPHs who are treating mental illnesses in South Africa. The results from this scoping review will help inform the subsequent phases of the study and contribute to the growing literature on suitable and effective models for collaborations between the traditional and Western health systems. This study will use publicly available materials and will not include human or animal participants; therefore, the study does not require ethical approval. The scoping report will be presented to other key health system stakeholders and will be introduced at conferences and other academic research gatherings. Findings will also be published in an open-access journal.

Correction notice This article has been corrected since it first published. The provenance and peer review statement has been included.

Acknowledgements The authors acknowledge Dr Tivani Mashamba-Thompson of the University of KwaZulu-Natal for consultation during the initial drafts of this scoping review protocol. We also thank Ms Karen Cook, a librarian at the University of the Western Cape, for providing guidance on the search terms to be applied in the study.

Contributors All authors contributed meaningfully to the preparation, drafting and editing of this scoping review protocol. NAJ (corresponding author) prepared the initial draft of this manuscript. UL guided the research objectives, the core research question and contributed to all the drafts of the manuscript. AN provided methodological guidance and contributed to shaping the overall manuscript. All authors approved the final submitted manuscript and agree to be accountable for all aspects of this protocol.

Funding This work is based on the research supported by the South African Research Chairs Initiative of the Department of Science and Technology and the
National Research Foundation of South Africa (Grant No. 98918). The authors would also like to acknowledge funding from the South African Medical Research Council and the Belgian Development Cooperation through the Institute of Tropical Medicine Antwerp.

Disclaimer Any opinion, finding, and conclusion or recommendation expressed in this material is that of the authors and not the funders.

Competing interests None declared.

Patient consent for publication Not required.

Provenance and peer review Not commissioned; externally peer reviewed.

Supplemental material This content has been supplied by the author(s). It has not been vetted by BMJ Publishing Group Limited (BMJ) and may not have been peer-reviewed. Any opinions or recommendations discussed are solely those of the author(s) and are not endorsed by BMJ. BMJ disclaims all liability and responsibility arising from any reliance placed on the content. Where the content includes any translated material, BMJ does not warrant the accuracy and reliability of the translations (including but not limited to local regulations, clinical guidelines, terminology, drug names and drug dosages), and is not responsible for any error and/or omissions arising from translation and adaptation or otherwise.

Open access This is an open access article distributed in accordance with the Creative Commons Attribution Non Commercial (CC BY-NC 4.0) license, which permits others to distribute, remix, adapt, build upon this work non-commercially, and license their derivative works on different terms, provided the original work is properly cited, appropriate credit is given, any changes made indicated, and the use is non-commercial. See: http://creativecommons.org/licenses/by-nc/4.0/.

ORCID iD

Ngcwalisa Amanda Jama http://orcid.org/0000-0003-4339-6220

\section{REFERENCES}

1 Latif SS. Integration of African traditional health practitioners and medicine into the health care management system in the province of Limpopo. Stellenbosch: University of Stellenbosch, 2010.

2 WHO. Mental health action plan 2013-2020, 2013.

3 Wreford J. Missing each other: problems and potential for collaborative efforts between biomedicine and traditional healers in South Africa in the time of AIDS. Soc Dyn 2005;31:55-89.

4 WHO. International conference on primary health care. Alma Ata, USSR: International Conference on Primary Health Care, 1978.

5 Campbell-Hall V, Petersen I, Bhana A, et al. Collaboration between traditional practitioners and primary health care staff in South Africa: developing a workable partnership for community mental health services. Transcult Psychiatry 2010;47:610-28.

6 Peu MD, Troskie R, Hattingh SP. The attitude of community health nurses towards integration of traditional healers in primary health care in north-west province. Curationis 2001;24:49-55.

7 Zhang X, Organization WH. Traditional medicine strategy 20022005 , 2002.

8 WHO. mhGAP operations manual: mental health Gap Action Programme (mhGAP). Geneva: World Health Organization, Back to cited text; 2018.

9 WHO. Global report on traditional and complementary medicine. Geneva, Switzerland, 2019.

10 Lee RP. Comparative studies of health care systems. Soc Sci Med 1982;16:629-42.

11 Pinkoane MG, Greeff M, Koen MP. Policy makers' perceptions and attitudes regarding incorporation of traditional healers into the national health care delivery system. Curationis 2008;31:4-12.

12 Busia K, Kasilo OM. Collaboration between traditional health practitioners and conventional health practitioners: some country experiences. Afr Health Monit 2010;13:40-6.

13 Burns JK, King H, Saloojee S. KwaZulu-Natal treatment protocols for mental health disorders. KwaZulu-Natal provincial directorate for health, 2007

14 Green EC. Can collaborative programs between biomedical and African indigenous health practitioners succeed? Soc Sci Med 1988;27:1125-30.

15 Gumede MV. Traditional healers: a medical practitioner's perspective. Skotaville, 1990

16 Patel V, Belkin G, Chockalingam A, et al. Integrating mental health services into priority health care platforms: addressing a grand challenge in global mental health. PLoS Med 2013;10:e1001448.

17 Gureje O, Nortje G, Makanjuola V, et al. The role of global traditional and complementary systems of medicine in the treatment of mental health disorders. Lancet Psychiatry 2015;2:168-77. 
18 Park YL, Canaway R. Integrating traditional and complementary medicine with national healthcare systems for universal health coverage in Asia and the Western Pacific. Health Syst Reform 2019:5:24-31.

19 Mokgobi MG. Health care practitioners' opinions about traditional healing. Afr J Phys Health Educ Recreat Dance 2014;20:14.

20 Schierenbeck I, Johansson P, Andersson LM, et al. Collaboration or renunciation? The role of traditional medicine in mental health care in Rwanda and Eastern Cape Province, South Africa. Glob Public Health 2018;13:159-72.

21 Davis K, Drey N, Gould D. What are scoping studies? A review of the nursing literature. Int J Nurs Stud 2009;46:1386-400.
22 Arksey H, O'Malley L. Scoping studies: towards a methodological framework. Int J Soc Res Methodol 2005;8:19-32.

23 Levac D, Colquhoun H, O'Brien KK. Scoping studies: advancing the methodology. Implement Sci 2010;5:69.

24 Peters M, Godfrey C, Khalil H, et al. 2017 Guidance for the Conduct of JBI Scoping Reviews, 2017.

25 Ouzzani M, Hammady H, Fedorowicz Z, et al. Rayyan - a web and mobile app for systematic reviews. Syst Rev 2016;5:210.

26 Tricco AC, Lillie E, Zarin W, et al. PRISMA extension for scoping reviews (PRISMA-ScR): checklist and explanation. Ann Intern Med 2018;169:467-73. 